\title{
Delayed hospital admission of patients with aneurysmal subarachnoid hemorrhage: clinical presentation, treatment strategies, and outcome
}

\author{
*Lukas Goertz, MD, ${ }^{1}$ Muriel Pflaeging, ${ }^{1}$ Christina Hamisch, MD, ${ }^{1}$ Christoph Kabbasch, MD, ${ }^{2}$ \\ Lenhard Pennig, MD, ${ }^{2}$ Niklas von Spreckelsen, MD, ${ }^{1,3}$ Kai Laukamp, MD, ${ }^{2,4}$ Marco Timmer, MD, ${ }^{1}$ \\ Roland Goldbrunner, MD, ${ }^{1}$ Gerrit Brinker, MD,1 and Boris Krischek, MD1

\begin{abstract}
1 University of Cologne, Medical Faculty and University Hospital, Center for Neurosurgery, and ${ }^{2}$ Institute for Diagnostic and Interventional Radiology, Medical Faculty and University Hospital, University of Cologne, Germany; ${ }^{3}$ Department of Neurosurgery, Harvey Cushing Neuro-Oncology Laboratories, Brigham and Women's Hospital, Harvard Medical School, Boston, Massachusetts; and ${ }^{4}$ Department of Radiology, University Hospitals Cleveland Medical Center, Cleveland, Ohio
\end{abstract}

OBJECTIVE Timely aneurysm occlusion and neurointensive care treatment are key principles in the management of aneurysmal subarachnoid hemorrhage (aSAH) to prevent secondary brain injury. Patients with early (EHA) and delayed hospital admission (DHA) were compared in terms of clinical presentation, treatment strategies, aSAH-related complications, and outcome.

METHODS In this retrospective study, consecutive aSAH patients were treated at a single neurovascular center between 2009 and 2019. Propensity score matching was performed to account for divergent baseline characteristics.

RESULTS Among 509 included patients, 55 were admitted more than 48 hours after ictus (DHA group). DHA patients were significantly younger ( $52 \pm 11$ vs $56 \pm 14$ years, $p=0.03$ ) and had lower World Federation of Neurosurgical Societies scores $(p<0.01$ ) than EHA patients. In $54.5 \%$ of the cases, DHA patients presented with neurological deterioration or aggravated symptoms. Propensity score matching revealed a higher vasospastic infarction rate in the DHA group $(41.5 \%)$ than in the EHA group $(22.6 \%)(p=0.04)$. A similar portion of patients in both groups achieved favorable outcome at midterm follow-up $(77.3 \%$ vs $73.6 \%, p=0.87)$. DHA patients $(62.3 \%)$ received conventional coiling more often than EHA patients $(41.5 \%)(p=0.03)$.

CONCLUSIONS DHA patients are at an increased risk of cerebral infarction. Nevertheless, state-of-the-art neurointensive care treatment can result in a good clinical outcome.

https://thejns.org/doi/abs/10.3171/2020.2.JNS20148

KEYWORDS cerebral infarction; clipping; coiling; delayed cerebral ischemia; modified Rankin Scale; propensity score; vascular disorders

A NEURYSMAL subarachnoid hemorrhage (aSAH) is caused by spontaneous rupture of intracranial aneurysms. The cardinal symptoms of aSAH include the sudden onset of severe headache, nausea, neck pain, and decreased levels of consciousness, with $20 \%$ of patients being comatose at initial presentation. ${ }^{1}$ aSAH represents a severe neurological condition, with mortality rates around $9 \%$ and permanent morbidity rates around $27 \%$, as reported by the ISAT study. ${ }^{1}$ Neurological outcome is highly dependent on early diagnosis and aggressive treatment at a neurovascular center. ${ }^{2}$ Immediate aneurysm embolization is required in order to minimize the risks of aneurysmal rebleeding, which occurs in $26 \%-73 \%$ of patients with untreated aneurysms during the hospital stay. ${ }^{3}$ Moreover, neurointensive care treatment is crucial-even in patients with mild symptoms-in order to reduce secondary brain damage, which is mostly related to cerebral vasospasm, brain swelling, and delayed cerebral ischemia. ${ }^{2}$

In the absence of the typical symptoms, aSAH may be misdiagnosed, which has been reported to occur in up to $51 \%$ of aSAH patients. ${ }^{4}$ Some studies evaluated the association of missed or delayed diagnosis of aSAH and

ABBREVIATIONS aSAH = aneurysmal subarachnoid hemorrhage CTA $=C T$ angiography; $\mathrm{DCl}=$ delayed cerebral ischemia; $\mathrm{DHA}=$ delayed hospital admission; $\mathrm{DSA}=$ digital subtraction angiography; EHA = early hospital admission; EVD = external ventricular drain; $m R S$ = modified Rankin Scale; WFNS = World Federation of Neurosurgical Societies.

SUBMITTED January 14, 2020. ACCEPTED February 19, 2020.

INCLUDE WHEN CITING Published online April 17, 2020; DOI: 10.3171/2020.2.JNS20148.

* L.G. and M.P. contributed equally to this work. 
functional outcome; however, most of these studies were performed in the pre-ISAT era. ${ }^{4-8}$ The misdiagnosis rates have decreased markedly over the last decades owing to the widespread availability and the increasing accuracy of neurovascular imaging. For instance, Inagawa reported that misdiagnosis rates dropped from $18 \%$ in the $1980 \mathrm{~s}$ to $9 \%$ in the 1990 s. ${ }^{6}$ It is probable that misdiagnosis rates have decreased even further since then.

Although misdiagnosis rates have dropped, some patients with mild or moderate symptoms of aSAH might not always seek medical advice immediately after symptom onset and may present several days after ictus when symptoms persist or become aggravated. ${ }^{6} \mathrm{We}$ hypothesize that these patients are at an increased risk of vasospasm, cerebral infarction, and poor outcome due to aneurysm rerupture and delayed intensive care treatment.

The objective of the present study was to compare clinical characteristics, treatment strategies, and clinical outcome between aSAH patients with early (EHA) and delayed hospital admission (DHA) who underwent a standardized aSAH treatment regimen at a neurovascular center. Additional propensity score matching was performed in order to account for potential differences between the two groups regarding the baseline characteristics.

\section{Methods \\ Study Population}

This is a retrospective study of consecutive patients who were admitted to our tertiary care neurovascular center (University Hospital of Cologne, Germany) for aSAH between January 2009 and July 2019. According to the statutes of our institutional guidelines, an ethics committee appraisal and informed consent for the collection, analysis, and publication of the retrospectively obtained and anonymized data were not required for conduction of this noninterventional study. Data analysis and reporting were performed in accordance with the STROBE guidelines.

\section{Inclusion Criteria}

All patients with the diagnosis of aSAH were considered for inclusion in this study. The patients were allocated to two groups, depending on whether they were admitted to the hospital within 24 hours after ictus (EHA) or later than 48 hours after ictus (DHA). The 48-hour threshold was selected on the basis that vasospasm typically occurs between day 3 and day 14 after initial aneurysm rupture. In some studies on vasospasm, such as the tirilazad mesylate and the Newton-2 studies, inclusion criteria included the need to have begun drug treatment within 48 hours after admission. ${ }^{9-11}$ We excluded patients who were admitted between 24 and 48 hours after ictus in order to increase selectivity. Further exclusion criteria were unclear date of ictus and inpatient treatment outside of a neurovascular center for $>24$ hours due to initial misdiagnosis.

\section{aSAH Management Protocol}

At initial presentation, patients routinely underwent native CT scanning of the head, CT angiography (CTA), and digital subtraction angiography (DSA) to determine the extent of the aSAH as well as aneurysm location, size, and morphology. Thereafter, the aneurysm embolization strategy was discussed within an interdisciplinary team of interventional neuroradiologists and vascular neurosurgeons, and allocation to a specific treatment modality was done in consensus. The antiplatelet regimen after intracranial stent implantation consisted of intravenous infusion of tirofiban started preinterventionally and continued for 24 hours, followed by 4 months of oral ingestion of clopidogrel and a lifelong oral administration of acetylsalicylic acid. ${ }^{12-15}$ After aneurysm treatment, the patients were routinely surveilled at an intensive care unit for at least 14 days after ictus. The blood flow velocity in the large cerebral arteries was determined daily by transcranial Doppler ultrasound. The onset of new neurological deficits, neurological deterioration, and blood flow velocities above $120 \mathrm{~cm} / \mathrm{sec}$ or an increase of $>50 \mathrm{~cm} / \mathrm{sec}$ within 24 hours were considered to be indicative of vasospasm. ${ }^{16}$ In this case, patients received a standard imaging protocol consisting of native CT, CTA, and CT perfusion studies. Patients with radiological proof of vasospasm underwent DSA with intraarterial administration of nimodipine and/ or balloon angioplasty of the spastic vessel segments. Upon proof of vasospasm, patients were sedated at least until the 14th day after ictus and the mean arterial pressure was elevated to $>100 \mathrm{~mm} \mathrm{Hg}$. Hypervolemia and hyperperfusion were not routinely applied. In patients without vasospasm, a native CT scan was acquired at least once every 7 days and before discharge. Insertion of an external ventricular drain (EVD) was done routinely upon radiological proof of hydrocephalus. Weaning of the EVD was performed on an individual basis, though at the earliest 14 days after ictus. In cases of chronic hydrocephalus, a ventriculoperitoneal shunt was implanted.

\section{Data Collection}

The following baseline parameters were collected from the medical records: patient demographics, date of ictus, date and time of hospital admission, initial symptoms, symptoms at admission, date of discharge, and neurological status. The neurological status was recorded at baseline, at discharge, and at follow-up visits, of which the first visit was generally between 3 and 6 months after ictus. Patients who were not available for follow-up were contacted by telephone. The World Federation of Neurosurgical Societies (WFNS) grading scale was used to determine the severity of aSAH. The extent of aSAH was evaluated by cranial CT using Fisher grades.

Functional outcome was determined at discharge and at the 3- to 6-month follow-up using the modified Rankin Scale (mRS). An mRS score of 0-2 was defined as a favorable outcome and a score of 3-6 as an unfavorable outcome, with a score of 6 indicating death.

For all patients, radiological reports and CT scans were reviewed in order to identify the occurrence of vasospasm and cerebral infarction. Vasospasm was defined as distinct narrowing of the cerebral arteries when compared to those shown on baseline CTA, as determined by an experienced consultant neuroradiologist. Cerebral infarction was defined as a new ischemic lesion on CT (or MRI in a minority of cases). The cause of cerebral infarction was discussed in each case, including vasospastic infarction, 
elevated intracranial pressure, mass effect, and procedurerelated infarctions. Both the overall infarction rate and the vasospastic infarction rate were reported in the current study.

The following interventions are reported in the current study: EVD placement, hemicraniectomy, surgical and endovascular aneurysm treatment, tracheotomy, and implantation of a ventriculoperitoneal shunt.

\section{Statistical Analysis}

Qualitative variables were expressed as numbers and percentages and compared using the chi-square or Fisher's exact test, when appropriate. Quantitative variables were presented as means \pm SD. Normal distribution of quantitative variables was assessed by the Shapiro-Wilk test. Groups were compared with the Student t-test (normally distributed data) and the Mann-Whitney U-test (nonnormally distributed data). In order to account for significant differences of baseline parameters between the groups, 1:1 matching was performed based on individual propensity scores. Propensity scores were calculated using a multivariate logistic regression model and the following covariates: patient age, sex, WFNS score, Fisher grade, presence of parenchymal hematoma, and presence of intraventricular hemorrhage. All calculations were performed using SPSS software (version 25.0, IBM Corp.). For propensity score analysis, the R 3.3.0 plug-in (https://cran.r-project. org/) was installed. A p value $<0.05$ was considered to be statistically significant.

\section{Results}

\section{Patient and Aneurysm Characteristics}

During the study period, a total of 561 patients with aSAH were admitted to our hospital (University Hospital of Cologne, Germany). Of those, 454 patients presented within 24 hours after ictus (EHA group) and 55 later than 48 hours after ictus (DHA group). We excluded 37 patients who were admitted between 24 and 48 hours after ictus, 11 patients who had an unclear date of ictus, and 4 patients who were treated at a peripheral hospital for more than 24 hours due to initial misdiagnosis.

Among 55 patients in the DHA group, the initial symptoms were head and neck pain in 53 patients $(96.4 \%)$, nausea in $6(10.9 \%)$, neurological deficits in $5(9.1 \%$, disturbance of consciousness in 2, hypesthesia in 1 , oculomotor palsy in 1, and aphasia in 1), and deterioration of the general condition in 4 (7.3\%). Of these 55 patients, $10(18.2 \%)$ presented as outpatients beforehand, but the correct diagnosis could not be established due to mild or atypical symptom presentation.

The patients in the DHA group were finally admitted to our institution for the following reasons: persisting symptoms in 25 cases $(45.5 \%)$, progressive symptoms in $15(27.3 \%)$, recurrent symptoms after initial improvement in 8 (14.5\%), and new neurological deficits in 7 (12.7\%). Among the patients with new neurological deficits, 4 had a hemiparesis, 2 had diplopia, and 1 had a seizure.

Five DHA patients (9.1\%) had angiographic proof of vasospasm on admission. Among those, 3 presented with severe headache and 2 with neurological deficits (hemi- paresis and dysarthria in both cases). All these patients developed vasospastic infarction. At clinical follow-up, 2 patients achieved a favorable outcome and 3 had an unfavorable outcome.

In the DHA group, the mean patient age was $51.8 \pm 10.8$ years, and $61.8 \%$ were female. At admission, the median WFNS score was I, and $29.1 \%$ had a Fisher grade 4 hemorrhage.

When compared to EHA patients, DHA patients were significantly younger $(\mathrm{p}=0.03)$, had a better median WFNS score $(p<0.01)$, and also had a lower median Fisher grade $(\mathrm{p}<0.01)$, as detailed in Table 1 .

To account for the significant differences in clinical and demographic parameters between the two groups, we performed a propensity score adjustment. After 1:1 propensity score matching, 53 DHA patients were compared with 53 EHA patients who had similar baseline characteristics, as shown in Table 1.

Baseline aneurysm characteristics are given in Table 2. The mean number of aneurysms per patient was 1.37 in the EHA group and 1.38 in the DHA group. A similar portion of patients had secondary aneurysms besides the ruptured aneurysm (EHA 23.1\%, DHA 27.3\%; p = 0.49). The mean aneurysm size was comparable between both groups (EHA $6.0 \pm 3.6 \mathrm{~mm}$, DHA $6.0 \pm 3.2 \mathrm{~mm}$; $=0.98$ ), while aneurysms in the EHA group had a significantly wider neck (EHA $3.5 \pm 1.8 \mathrm{~mm}$, DHA $2.9 \pm 1.4 \mathrm{~mm}$; p $<0.01)$. After accounting for the propensity scores, there were no significant differences among baseline aneurysm characteristics (Table 2).

\section{Treatment}

A list of interventions is given in Table 3. EVD placement due to initial hydrocephalus was performed significantly more often in EHA patients (75.8\%) than in DHA patients $(43.6 \%)(\mathrm{p}<0.01)$, while there was no significant difference regarding hemicraniectomy rates $(\mathrm{p}=0.57)$. Tracheotomy was more often required in patients in the EHA group (40.1\%) than in the DHA group (20.0\%) (p $<0.01$ ). DHA patients were significantly more frequently allocated to receive endovascular coiling $(60.0 \%)$ than EHA patients $(39.0 \%, \mathrm{p}<0.01)$, while microsurgical clipping and stent-assisted procedures were more commonly performed in EHA patients without attaining statistical significance (Table 3). After propensity score adjustment, EVDs were placed by trend more often in EHA patients $(p=0.08)$, while there were no significant differences regarding hemicraniectomy and tracheotomy rates (Table 3). Furthermore, propensity score matching confirmed that endovascular coiling was significantly more common among DHA patients than among EHA patients $(62.3 \%$ vs $41.5 \%)(\mathrm{p}=0.03)$.

\section{aSAH-Related Complications}

aSAH-related complications are listed in Table 4. In the DHA group, vasospasm occurred in $54.5 \%$, which was comparable to the overall cohort of the EHA group $(44.7 \%, \mathrm{p}=0.17)$. The overall cerebral infarction rate was significantly higher among DHA patients (49.1\%) than among EHA patients $(33.9 \%)(\mathrm{p}=0.03)$. Vasospastic infarction was more frequent in DHA patients $(41.8 \%)$ than 
TABLE 1. Baseline clinical and demographic characteristics in the overall and 1:1 propensity score-matched cohorts

\begin{tabular}{|c|c|c|c|c|c|c|}
\hline \multirow[b]{2}{*}{ Parameter } & \multirow{2}{*}{$\begin{array}{c}\text { EHA } \\
(n=454)\end{array}$} & \multirow{2}{*}{$\begin{array}{c}\text { DHA } \\
(n=55)\end{array}$} & \multirow{2}{*}{$\begin{array}{c}p \\
\text { Value }\end{array}$} & \multicolumn{3}{|c|}{ Propensity Score Matching } \\
\hline & & & & $\mathrm{EHA}(n=53)$ & $\mathrm{DHA}(\mathrm{n}=53)$ & p Value \\
\hline Patient age, yrs & $56.0 \pm 14.0$ & $51.8 \pm 10.8$ & 0.03 & $53.4 \pm 11.9$ & $51.5 \pm 10.9$ & 0.39 \\
\hline Sex & & & 0.28 & & & 0.54 \\
\hline Female & $313(68.9 \%)$ & $34(61.8 \%)$ & & $36(67.9 \%)$ & $33(62.3 \%)$ & \\
\hline Male & $141(31.1 \%)$ & $21(38.2 \%)$ & & $17(32.1 \%)$ & $20(37.7 \%)$ & \\
\hline WFNS score & & & $<0.01$ & & & 0.82 \\
\hline I & $103(22.7 \%)$ & $37(67.3 \%)$ & & $36(67.9 \%)$ & $35(66.0 \%)$ & \\
\hline II & $70(15.4 \%)$ & $10(18.2 \%)$ & & $11(20.8 \%)$ & $10(18.9 \%)$ & \\
\hline III & $59(13.0 \%)$ & $5(9.1 \%)$ & & $2(3.8 \%)$ & $5(9.4 \%)$ & \\
\hline IV & $57(12.6 \%)$ & $2(3.6 \%)$ & & $3(5.7 \%)$ & $2(3.8 \%)$ & \\
\hline V & $165(36.3 \%)$ & $1(1.8 \%)$ & & $1(1.9 \%)$ & $1(1.9 \%)$ & \\
\hline Fisher grade & & & $<0.01$ & & & 0.99 \\
\hline 1 & $5(1.1 \%)$ & $4(7.3 \%)$ & & $2(3.8 \%)$ & $2(3.8 \%)$ & \\
\hline 2 & $19(4.2 \%)$ & $15(27.3 \%)$ & & $15(28.3 \%)$ & $15(28.3 \%)$ & \\
\hline 3 & $172(37.9 \%)$ & $20(36.4 \%)$ & & $20(37.7 \%)$ & $20(37.7 \%)$ & \\
\hline 4 & $258(56.8 \%)$ & $16(29.1 \%)$ & & $16(30.2 \%)$ & $16(30.2 \%)$ & \\
\hline Parenchymal hemorrhage & $139(30.6 \%)$ & $7(12.7 \%)$ & $<0.01$ & $8(15.1 \%)$ & $7(13.2 \%)$ & 0.78 \\
\hline Intraventricular hemorrhage & $199(43.8 \%)$ & $12(21.8 \%)$ & $<0.01$ & $14(26.4 \%)$ & $12(22.6 \%)$ & 0.65 \\
\hline
\end{tabular}

Values are presented as the number (\%) of patients unless otherwise indicated.

in EHA patients $(27.8 \%)(\mathrm{p}=0.03)$. Chronic hydrocephalus requiring permanent cerebrospinal fluid drainage was by trend more common in the EHA group (27.1\%) than in the DHA group $(16.4 \%)(\mathrm{p}=0.09)$.

In the propensity score-matched cohorts, vasospasm occurred slightly more often in DHA patients $(56.6 \%$ vs $41.5 \%)$ without reaching statistical significance $(\mathrm{p}=0.12)$. However, propensity score matching confirmed a significantly higher infarction rate in the DHA group (49.1\%) than in the EHA group $(26.4 \%)(p=0.02)$, while chronic hydrocephalus rates were not significantly different $(\mathrm{p}=$ 0.79).

\section{Clinical Outcome}

Clinical outcome is detailed in Table 5. The in-hospital mortality rate was significantly higher for EHA patients $(26.4 \%)$ than for DHA patients $(5.5 \%)(\mathrm{p}<0.01)$. At discharge, favorable outcome was more often achieved in the DHA group (67.3\%) than in the EHA group (29.7\%) (p < 0.01 ). At the 3- to 6-month follow-up visit, favorable outcome was obtained in $78.2 \%$ of DHA patients, compared to $43.8 \%$ of EHA patients $(\mathrm{p}<0.01)$.

After 1:1 propensity score matching, mortality rates and clinical outcome were comparable between the two groups, as detailed in Table 5.

TABLE 2. Baseline aneurysm characteristics in the overall and 1:1 propensity score-matched cohorts

\begin{tabular}{lcccccc}
\hline & & & \multicolumn{3}{c}{ Propensity Score Matching } \\
\cline { 5 - 7 } \multicolumn{1}{c}{ Parameter } & EHA $(\mathrm{n}=454)$ & $\mathrm{DHA}(\mathrm{n}=55)$ & $\mathrm{p}$ Value & $\mathrm{EHA}(\mathrm{n}=53)$ & $\mathrm{DHA}(\mathrm{n}=53)$ & $\mathrm{p}$ Value \\
\hline No. of aneurysms & & & 0.23 & & 0.78 \\
\hline 1 & $349(76.9 \%)$ & $40(72.7 \%)$ & & $44(83.0 \%)$ & $39(73.6 \%)$ & \\
\hline 2 & $59(13.0 \%)$ & $12(21.8 \%)$ & & $6(11.3 \%)$ & $11(20.8 \%)$ & \\
\hline 3 & $34(7.5 \%)$ & $1(1.8 \%)$ & & $1(1.9 \%)$ & $1(1.9 \%)$ & \\
\hline 4 & $8(1.8 \%)$ & $1(1.8 \%)$ & & $1(1.9 \%)$ & $1(1.9 \%)$ & \\
\hline 5 & $4(0.9 \%)$ & $1(1.8 \%)$ & & $1(1.9 \%)$ & $1(1.9 \%)$ & \\
\hline Multiple aneurysms & $105(23.1 \%)$ & $15(27.3 \%)$ & 0.49 & $9(17.0 \%)$ & $14(26.4 \%)$ & 0.24 \\
\hline Aneurysm size $(\mathrm{mm})$ & $6.0 \pm 3.6$ & $6.0 \pm 3.2$ & 0.98 & $5.7 \pm 3.2$ & $5.9 \pm 3.3$ & 0.76 \\
\hline Neck width $(\mathrm{mm})$ & $3.5 \pm 1.8$ & $2.9 \pm 1.4$ & $<0.01$ & $3.1 \pm 1.3$ & $2.8 \pm 1.2$ & 0.23 \\
\hline
\end{tabular}

Values are presented as the number $(\%)$ of patients or as the mean \pm SD unless otherwise indicated. 
TABLE 3. Interventions among EHA and DHA patients in the overall and 1:1 propensity score-matched cohorts

\begin{tabular}{lcrrrrr}
\hline & & & \multicolumn{3}{c}{ Propensity Score Matching } \\
\cline { 5 - 7 } \multicolumn{1}{c}{ Parameter } & EHA $(n=454)$ & DHA $(n=55)$ & $p$ Value & EHA $(n=53)$ & DHA $(n=53)$ & $p$ Value \\
\hline EVD & $344(75.8 \%)$ & $24(43.6 \%)$ & $<0.01$ & $33(62.3 \%)$ & $24(45.3 \%)$ & 0.08 \\
\hline Hemicraniectomy & $62(13.7 \%)$ & $6(10.9 \%)$ & 0.57 & $6(11.3 \%)$ & $6(11.3 \%)$ & 0.99 \\
\hline Tracheotomy & $182(40.1 \%)$ & $11(20.0 \%)$ & $<0.01$ & $14(26.4 \%)$ & $11(20.8 \%)$ & 0.49 \\
\hline Aneurysm Tx & & & & & \\
\hline Conservative & $34(7.5 \%)$ & $1(1.8 \%)$ & 0.16 & $1(1.9 \%)$ & $1(1.9 \%)$ & 0.99 \\
\hline Clipping & $164(36.1 \%)$ & $15(27.3 \%)$ & 0.19 & $21(39.6 \%)$ & $13(24.5 \%)$ & 0.10 \\
\hline Coiling & $177(39.0 \%)$ & $33(60.0 \%)$ & $<0.01$ & $22(41.5 \%)$ & $33(62.3 \%)$ & 0.03 \\
\hline Stent/flow diversion & $78(17.2 \%)$ & $6(10.9 \%)$ & 0.24 & $9(17.0 \%)$ & $6(11.3 \%)$ & 0.40 \\
\hline
\end{tabular}

Tx = treatment.

Values are presented as the number (\%) of patients unless otherwise indicated.

\section{Discussion}

In the current study, we compared clinical characteristics, SAH-related complications, and clinical outcomes between SAH patients who had early and those who had delayed admissions ( $>48$ hours after ictus) to a specialized neurovascular center and who received standardized neurointensive care treatment.

As a major finding, patients in the DHA group typically had a low-grade aSAH with only mild to moderate symptoms, as reflected by lower WFNS scores, than patients in the EHA group. In fact, only $12.7 \%$ of DHA patients had neurological symptoms when they presented to our neurovascular center $>48$ hours after ictus. In comparison, $61.9 \%$ of EHA patients presented with a WFNS score between III and V, which is characterized by a varying extent of neurological deterioration. Patients with deteriorated consciousness routinely receive a CT scan immediately after hospital admission, hence carrying a low risk of being misdiagnosed.

In the DHA group, $18.2 \%$ sought immediate medical advice as outpatients; however, the correct diagnosis was not established, possibly due to mild symptoms.

Some studies addressed the outcome and risk factors of patients with delayed hospital admission due to misdiagnosis after aSAH. Inagawa reported that misdiagnosis occurs typically in patients with WFNS scores of I and II, ${ }^{6}$ which is confirmed by other studies and by our data. ${ }^{8,17,18}$ In the overall study population, misdiagnosed patients had a better outcome than patients with an EHA, as it was the case in our study. ${ }^{6}$ However, a direct comparison of DHA with EHA patients is confounded by significant differences in baseline characteristics, especially regarding WFNS grade. To counteract this selection bias, Kowalski et al. performed a subgroup analysis of patients with Hunt and Hess grades I and II and reported significantly higher mortality and disability rates among misdiagnosed patients (22\% vs $8 \%$ ). ${ }^{3}$ Likewise, Mayer et al. reported favorable outcomes in $91 \%$ of patients with a correct diagnosis versus $53 \%$ of those with a delayed diagnosis. ${ }^{8}$ Although these studies suggest misdiagnosis as a prognostic factor for patient outcome, the impact of DHA has not yet been investigated systematically by a matched case-control study. Moreover, the patient groups with misdiagnosis in the literature cannot be compared to our DHA group directly, as it may exclude patients who had rebleeding with poor outcome or death prior to hospital admission. In addition, most available studies on DHA were performed in the pre-ISAT era, and hence, the results may not directly apply to results obtained using contemporary, state-of-the-art aSAH treatment. DHA patients were typically in good overall condition and only had mild to moderate symptoms, which predominantly translated into WFNS grades I and II. For that reason, the DHA group cannot be compared with the overall aSAH cohort directly. To the best of our knowledge, there is no study available that systematically analyzed outcomes of DHA patients by adjusting for confounding variables.

TABLE 4. aSAH-related adverse events in the overall and 1:1 propensity score-matched cohorts

\begin{tabular}{lcrrrrr}
\hline & & & & \multicolumn{3}{c}{ Propensity Score Matching } \\
\cline { 5 - 7 } \multicolumn{1}{c}{ Parameter } & EHA $(\mathrm{n}=454)$ & $\mathrm{DHA}(\mathrm{n}=55)$ & $\mathrm{p}$ Value & $\mathrm{EHA}(\mathrm{n}=53)$ & $\mathrm{DHA}(\mathrm{n}=53)$ & $\mathrm{p}$ Value \\
\hline Vasospasm & $203(44.7 \%)$ & $30(54.5 \%)$ & 0.17 & $22(41.5 \%)$ & $30(56.6 \%)$ & 0.12 \\
\hline Cerebral infarction & $154(33.9 \%)$ & $27(49.1 \%)$ & 0.03 & $14(26.4 \%)$ & $26(49.1 \%)$ & 0.02 \\
\hline Vasospastic infarction & $126(27.8 \%)$ & $23(41.8 \%)$ & 0.03 & $12(22.6 \%)$ & $22(41.5 \%)$ & 0.04 \\
\hline Chronic hydrocephalus & $123(27.1 \%)$ & $9(16.4 \%)$ & 0.09 & $8(15.1 \%)$ & $9(17.0 \%)$ & 0.79 \\
\hline
\end{tabular}

Values are presented as the number (\%) of patients unless otherwise indicated. 
TABLE 5. Clinical outcome as determined by $m R S$ score in the overall and 1:1 propensity scorematched cohorts

\begin{tabular}{|c|c|c|c|c|c|c|}
\hline \multirow[b]{2}{*}{ Parameter } & \multirow[b]{2}{*}{$\mathrm{EHA}(\mathrm{n}=454)$} & \multirow[b]{2}{*}{$\mathrm{DHA}(\mathrm{n}=55)$} & \multirow[b]{2}{*}{ p Value } & \multicolumn{3}{|c|}{ Propensity Score Matching } \\
\hline & & & & $\mathrm{EHA}(n=53)$ & $\mathrm{DHA}(\mathrm{n}=53)$ & $p$ Value \\
\hline Length of stay, days & $29.2 \pm 20.5$ & $25.8 \pm 16.5$ & 0.17 & $27.3 \pm 15.5$ & $26.3 \pm 16.7$ & 0.75 \\
\hline mRS score at discharge & & & $<0.01$ & & & 0.71 \\
\hline 0 & $66(14.5 \%)$ & $24(43.6 \%)$ & & $18(34.0 \%)$ & $23(43.3 \%)$ & \\
\hline 1 & $38(8.4 \%)$ & $5(9.1 \%)$ & & $9(17.0 \%)$ & $5(9.4 \%)$ & \\
\hline 2 & $31(6.8 \%)$ & $8(14.5 \%)$ & & $4(7.5 \%)$ & $7(13.2 \%)$ & \\
\hline 3 & $39(8.6 \%)$ & $4(7.3 \%)$ & & $4(7.5 \%)$ & $4(7.5 \%)$ & \\
\hline 4 & $101(22.2 \%)$ & $9(16.4 \%)$ & & $9(17.0 \%)$ & $9(17.0 \%)$ & \\
\hline 5 & $59(13.0 \%)$ & $2(3.6 \%)$ & & $3(5.7 \%)$ & $2(3.8 \%)$ & \\
\hline 6 & $120(26.4 \%)$ & $3(5.5 \%)$ & & $6(11.3 \%)$ & $3(5.7 \%)$ & \\
\hline $\mathrm{mRS}$ at 6-mo FU & & & $<0.01$ & & & 0.87 \\
\hline 0 & $129(28.4 \%)$ & $28(50.9 \%)$ & & $29(54.7 \%)$ & 27 (50.9\%) & \\
\hline 1 & $45(9.9 \%)$ & $11(20.0 \%)$ & & $7(13.2 \%)$ & $10(18.9 \%)$ & \\
\hline 2 & $25(5.5 \%)$ & $4(7.3 \%)$ & & $3(5.7 \%)$ & $4(7.5 \%)$ & \\
\hline 3 & $31(6.8 \%)$ & $2(3.6 \%)$ & & $2(3.8 \%)$ & $2(3.8 \%)$ & \\
\hline 4 & $60(13.2 \%)$ & $6(10.9 \%)$ & & $4(7.5 \%)$ & $6(11.3 \%)$ & \\
\hline 5 & $43(9.5 \%)$ & $1(1.8 \%)$ & & $2(3.8 \%)$ & $1(1.9 \%)$ & \\
\hline 6 & $121(26.7 \%)$ & $3(5.5 \%)$ & & $6(11.3 \%)$ & $3(5.7 \%)$ & \\
\hline
\end{tabular}

To overcome these limitations, we performed a 1:1 propensity score matching of EHA and DHA patients who underwent a standardized aSAH treatment protocol at our tertiary care hospital. After the matching, the study groups predominantly contained younger patients with low WFNS grades.

\section{Complications}

The overall neurological outcome of aSAH patients mainly depends on the extent of primary and secondary brain damage, which is mainly related to mass effect, aneurysm rebleeding, vasospasm, and delayed cerebral ischemia. ${ }^{16,19-21}$ Particularly, the risk for aneurysmal rebleeding is highest within 6 hours after ictus and decreases thereafter. ${ }^{22}$ In contrast, the risk of vasospasm increases steeply at the 3rd day after aneurysm rupture and peaks after 1 week. ${ }^{23}$ Recognition of the signs and symptoms of aSAH, early aneurysm embolization, and treatment within a neurovascular center are considered as the key principles in aSAH management in order to limit the extent of primary brain damage and to decrease the risk of secondary brain damage. ${ }^{2}$

In our cohort, $54.5 \%$ of DHA patients were finally admitted due to neurological deterioration or aggravated symptoms. In this context, Miyazaki et al. reported that the neurological status of aSAH patients is significantly worsened when they are readmitted due to initial misdiagnosis. ${ }^{17}$ Likewise, in the study by Mayer et al., $48 \%$ of patients experienced rebleeding or deterioration until readmission, which is generally considered as a poor prognostic factor. ${ }^{8,24}$
Prompted by several cases of cerebral vasospasm and delayed cerebral ischemia of aSAH patients with delayed admission to our institution, we questioned whether these patients might have an increased risk of cerebral infarction and unfavorable outcome when directly compared to EHA patients with similar baseline characteristics. Although only $9.1 \%$ of DHA patients had angiographic proof of vasospasm on baseline angiography, they had a significantly higher rate of vasospastic infarction (41.5\%) than EHA patients $(22.6 \%)$ in the matched analysis. This finding correlates with an overall increased frequency of vasospasm in the DHA group (56.6\% vs $41.5 \%$ ). Although evidencebased treatment strategies of vasospasm are sparse, mild to moderate hypertension and administration of oral nimodipine has been widely adopted in order to prevent and treat vasospasm. ${ }^{25}$ Intraarterial infusion of vasodilating agents and angioplasty represent further therapeutic options to relieve the spasm. ${ }^{26}$ Our data clearly support the concept of ultra-early antivasospastic treatment from the 1st day of ictus in order to minimize the risk of cerebral infarction..$^{23}$ In particular, state-of-the-art-and potentially prolonged-neurointensive care treatment is of utmost importance for DHA patients, as they are prone to vasospasm and infarction.

\section{Clinical Outcome}

Interestingly, the increased infarction rates among DHA patients did not translate into a worse functional outcome, although delayed cerebral ischemia represents a major risk factor for case fatality and morbidity after aSAH. ${ }^{19-21}$ The overall mortality rate among DHA patients 
was $5.7 \%$, and $78.2 \%$ could live independently $3-6$ months after ictus, which is comparable to the rate seen in the matched EHA group. On the one hand, these findings suggest that neurointensive care can lead to good functional outcome of aSAH patients, even when started in a delayed fashion. On the other hand, the various clinical outcome domains may not be fully reflected by the mRS score that was determined retrospectively in our study. The mRS score measures the degree of disability or dependence in daily activities. However, although long-term survivors of aSAH can regain independence in basic activities of daily living, many have persistent deficits across multiple cognitive domains, which can be related to cerebral infarction. ${ }^{27,28}$ Cognitive function is not adequately addressed by the $\mathrm{mRS}$, and it is difficult to determine retrospectively. Hence, it may be speculated that the higher infarction rates among DHA patients may be associated with more severe cognitive impairment, when compared to EHA patients. However, this hypothesis cannot be confirmed by the results of our retrospective analysis and needs further evaluation.

\section{Treatment Strategy}

The majority of DHA patients were treated by endovascular coiling. Since microsurgical clipping and intracranial stent implantation are associated with a varying degree of vessel reconstruction, these techniques were avoided when the vessels showed signs of vasospasm. Our main concern was that hemodynamics in vasospastic segments might be further impeded by vessel reconstruction, hence provoking cerebral hypoperfusion. In aneurysms with challenging anatomy for endovascular coiling, we pursued two strategies on a case-by-case basis: If possible, the rupture site of the aneurysm was covered with coils without targeting complete aneurysm occlusion. Thereafter, definite treatment by stent-assisted procedures or microsurgical clipping was performed after the vasospasm phase. If provisional coiling was not feasible, we considered treating the aneurysm after radiological improvement of the vasospasms. However, the best aneurysm embolization strategies for aSAH patients with vasospasm remain unclear and require further investigation by other studies.

\section{Limitations}

The presented study has several inherent limitations due to its retrospective, single-center design. The EHA and DHA groups were not balanced with regard to baseline characteristics. In order to mitigate this limitation-at least in part-we performed a matched analysis based on the individual propensity scores. Functional outcome was only assessed using the mRS, which lacks several outcome domains such as cognitive function. Moreover, we do not provide an analysis of complications and angiographic outcome of aneurysm treatment, which is outside of the scope of this paper.

With the aforementioned limitations in mind, the current study gives insight into the clinical characteristics of DHA patients and highlights the importance of early and aggressive neurointensive care treatment of aSAH patients in order to avoid vasospasm-related complications.

\section{Conclusions}

DHA patients are typically associated with low-grade SAH. In 54.5\%, the patients presented due to neurological deterioration or aggravating symptoms. Matched analysis revealed that DHA patients were more susceptible to cerebral infarction than EHA patients. However, favorable outcome was attained by a similar portion in both groups. The study highlights the importance of state-of-the-art neurointensive care treatment in order to minimize vasospasm-related complications. Nonetheless, a good clinical outcome can be obtained, even in case of a delayed admission to the hospital.

\section{References}

1. Molyneux A, Kerr R, Stratton I, et al. International Subarachnoid Aneurysm Trial (ISAT) of neurosurgical clipping versus endovascular coiling in 2143 patients with ruptured intracranial aneurysms: a randomised trial. Lancet. 2002;360(9342):1267-1274.

2. van Gijn J, Rinkel GJ. Subarachnoid haemorrhage: diagnosis, causes and management. Brain. 2001;124(Pt 2):249-278.

3. Kowalski RG, Claassen J, Kreiter KT, et al. Initial misdiagnosis and outcome after subarachnoid hemorrhage. JAMA. 2004;291(7):866-869.

4. Schievink WI, van der Werf DJ, Hageman LM, Dreissen JJ. Referral pattern of patients with aneurysmal subarachnoid hemorrhage. Surg Neurol. 1988;29(5):367-371.

5. Chan BS, Dorsch NW. Delayed diagnosis in subarachnoid haemorrhage. Med J Aust. 1991;154(8):509-511.

6. Inagawa T. Delayed diagnosis of aneurysmal subarachnoid hemorrhage in patients: a community-based study. J Neurosurg. 2011;115(4):707-714.

7. Kassell NF, Kongable GL, Torner JC, et al. Delay in referral of patients with ruptured aneurysms to neurosurgical attention. Stroke. 1985;16(4):587-590.

8. Mayer PL, Awad IA, Todor R, et al. Misdiagnosis of symptomatic cerebral aneurysm. Prevalence and correlation with outcome at four institutions. Stroke. 1996;27(9):1558-1563.

9. Haley EC Jr, Kassell NF, Apperson-Hansen C, et al. A randomized, double-blind, vehicle-controlled trial of tirilazad mesylate in patients with aneurysmal subarachnoid hemorrhage: a cooperative study in North America. J Neurosurg. 1997;86(3):467-474.

10. Hänggi D, Etminan N, Mayer SA, et al. Clinical trial protocol: phase 3, multicenter, randomized, double-blind, placebocontrolled, parallel-group, efficacy, and safety study comparing EG-1962 to standard of care oral nimodipine in adults with aneurysmal subarachnoid hemorrhage [NEWTON-2 (Nimodipine Microparticles to Enhance Recovery While Reducing TOxicity After SubarachNoid Hemorrhage)]. Neurocrit Care. 2019;30(1):88-97.

11. Kassell NF, Haley EC Jr, Apperson-Hansen C, Alves WM. Randomized, double-blind, vehicle-controlled trial of tirilazad mesylate in patients with aneurysmal subarachnoid hemorrhage: a cooperative study in Europe, Australia, and New Zealand. J Neurosurg. 1996;84(2):221-228.

12. Goertz L, Dorn F, Kraus B, et al. Improved occlusion rate of intracranial aneurysms treated with the Derivo embolization device: one-year clinical and angiographic follow-up in a multicenter study. World Neurosurg. 2019;126:e1503-e1509.

13. Goertz L, Dorn F, Kraus B, et al. Safety and efficacy of the Derivo Embolization Device for the treatment of ruptured intracranial aneurysms. J Neurointerv Surg. 2019;11(3):290295.

14. Goertz L, Dorn F, Siebert E, et al. Safety and efficacy of the Neuroform Atlas for stent-assisted coiling of intracra- 
nial aneurysms: a multicenter experience. J Clin Neurosci. 2019;68:86-91.

15. Goertz L, Smyk MA, Mpotsaris A, et al. Long-term angiographic results of the low-profile Acandis Acclino stent for treatment of intracranial aneurysms: a multicenter study [published online November 15, 2019]. Clin Neuroradiol. doi:10.1007/s00062-019-00847-4

16. Goertz L, Hamisch C, Kabbasch C, et al. Impact of aneurysm shape and neck configuration on cerebral infarction during microsurgical clipping of intracranial aneurysms [published online April 12, 2019]. J Neurosurg. doi:10.3171/2019.1.JNS183193

17. Miyazaki T, Ohta F, Moritake K, et al. The key to improving prognosis for aneurysmal subarachnoid hemorrhage remains in the pre-hospitalization period. Surg Neurol. 2006;65(4):360-366.

18. Vermeulen MJ, Schull MJ. Missed diagnosis of subarachnoid hemorrhage in the emergency department. Stroke. 2007;38(4):1216-1221.

19. Dankbaar JW, Rijsdijk M, van der Schaaf IC, et al. Relationship between vasospasm, cerebral perfusion, and delayed cerebral ischemia after aneurysmal subarachnoid hemorrhage. Neuroradiology. 2009;51(12):813-819.

20. Etminan N, Vergouwen MD, Ilodigwe D, Macdonald RL. Effect of pharmaceutical treatment on vasospasm, delayed cerebral ischemia, and clinical outcome in patients with aneurysmal subarachnoid hemorrhage: a systematic review and meta-analysis. J Cereb Blood Flow Metab. 2011;31(6):14431451.

21. Vergouwen MD, Vermeulen M, van Gijn J, et al. Definition of delayed cerebral ischemia after aneurysmal subarachnoid hemorrhage as an outcome event in clinical trials and observational studies: proposal of a multidisciplinary research group. Stroke. 2010;41(10):2391-2395.

22. Inagawa $T$. Ultra-early rebleeding within six hours after aneurysmal rupture. Surg Neurol. 1994;42(2):130-134.

23. Phan K, Moore JM, Griessenauer CJ, et al. Ultra-early angiographic vasospasm after aneurysmal subarachnoid hemorrhage: a systematic review and meta-analysis. World Neurosurg. 2017;102:632-638.e1.

24. Naidech AM, Janjua N, Kreiter KT, et al. Predictors and impact of aneurysm rebleeding after subarachnoid hemorrhage. Arch Neurol. 2005;62(3):410-416.
25. Dankbaar JW, Slooter AJ, Rinkel GJ, Schaaf IC. Effect of different components of triple-H therapy on cerebral perfusion in patients with aneurysmal subarachnoid haemorrhage: a systematic review. Crit Care. 2010;14(1):R23.

26. Hoh BL, Ogilvy CS. Endovascular treatment of cerebral vasospasm: transluminal balloon angioplasty, intra-arterial papaverine, and intra-arterial nicardipine. Neurosurg Clin $N$ Am. 2005;16(3):501-516, vi.

27. Al-Khindi T, Macdonald RL, Schweizer TA. Cognitive and functional outcome after aneurysmal subarachnoid hemorrhage. Stroke. 2010;41(8):e519-e536.

28. Huenges Wajer IM, Cremers CH, van Zandvoort MJ, et al. $\mathrm{CT}$ perfusion on admission and cognitive functioning 3 months after aneurysmal subarachnoid haemorrhage. $\mathrm{J} \mathrm{Neu}$ rol. 2015;262(3):623-628.

\section{Disclosures}

Dr. Kabbasch reports being a consultant for MicroVention and Acandis.

\section{Author Contributions}

Conception and design: Goertz, Pflaeging, von Spreckelsen, Brinker, Krischek. Acquisition of data: Goertz, Pflaeging, Hamisch, Pennig, Laukamp. Analysis and interpretation of data: Goertz, Pflaeging, Kabbasch, von Spreckelsen, Timmer, Krischek. Drafting the article: Goertz, Pflaeging, Brinker, Krischek. Critically revising the article: Goertz, Hamisch, Kabbasch, Pennig, von Spreckelsen, Laukamp, Timmer, Goldbrunner, Brinker, Krischek. Reviewed submitted version of manuscript: Goertz, Hamisch, Kabbasch, Pennig, von Spreckelsen, Laukamp, Timmer, Goldbrunner, Brinker, Krischek. Approved the final version of the manuscript on behalf of all authors: Goertz. Statistical analysis: Goertz, Pflaeging, Pennig, von Spreckelsen. Administrative/technical/material support: Timmer, Goldbrunner, Krischek.

\section{Correspondence}

Lukas Goertz: Center for Neurosurgery, University Hospital of Cologne, Germany. lukas.goertz@uk-koeln.de. 